\title{
Judgments of average magnitude: Analyses in terms of the functional measurement and two-stage models
}

\author{
DWIGHT W. CURTIS and LOIS C. MULLIN \\ University of Alberta, Edmonton, Alberta, Canada T6G 2E9
}

\begin{abstract}
Subjects made magnitude estimates of the average loudness of pairs of $1,000-\mathrm{Hz}$ tones varying in sound pressure. A test of fit of an averaging model employing an analysis of variance suggested that the judgments were internally consistent. However, estimates of the parameters of a two-stage model based on the assumption that power transformations were imposed in both input and output implied a nonlinear output function, inconsistent with the averaging model. Additional analyses employing a nonmetric scaling solution also suggested that output was nonlinear, indicating that this implication was not an artifact of the strong assumptions of the two-stage model. Large differences were found among the output functions of individual subjects, and it was suggested that these may have inflated the error term in the analysis of variance, reducing its power to detect violations of the additive model. Similar analyses were performed on data from judgments of average grayness collected by Weiss (1972).
\end{abstract}

N. H. Anderson and his associates have investigated the use of judgments of average magnitude with a simple linear model as a basis for scaling perceptual attributes. For an experiment in which subjects judge the average magnitude of pairs of stimuli, the averaging model is given by the expression

$$
\mathrm{J}_{\mathrm{ij}}=\mathrm{w}_{1} \mathrm{~V}_{\mathrm{i}}+\mathrm{w}_{2} \mathrm{~V}_{\mathrm{j}} \text {, }
$$

where $J$ is a judgment of the average magnitude of the $i$-th and $j$-th stimuli, $V_{i}$ and $V_{j}$ are scale values associated with the stimuli, and $w_{1}$ and $w_{2}$ reflect the relative weights assigned by the subject to the first and second stimuli in making his judgment. In an averaging model, $\mathrm{w}_{1}+\mathrm{w}_{2}=1$. If stimulus pairs are selected from a factorial design, the model may be tested empirically by an analysis of variance. Additivity implies that the interaction should be nonsignificant. If additivity holds, the marginal means constitute a subjective interval scale of the stimulus attribute. Anderson (1970a) has labeled this scaling solution "functional measurement."

In several experiments, Anderson $(1967,1970 \mathrm{~b}$, 1974) has obtained evidence that the averaging model holds when graphic ratings or category ratings are used to represent judgments. Since scales constructed by magnitude estimation procedures are nonlinear

The loudness data were from a thesis by the second author under the supervision of the first author, submitted to the Department of Psychology. California State University. Fullerton, in partial fultillment of the requirements of the MA degree. This research was supported by a grant from the California State University Foundation and by Grant A9582 from the National Research Council of Canada. Requests for reprints should be sent to Dwight W. Curtis, whose address is Department of Psychology, University of Alberta, Edmonton, Alberta, Canada T6G 2 E9. with category and graphic scales, he has argued that the magnitude estimation scales must be biased. This inference was directly tested by Weiss (1972) in a study in which both magnitude estimation and graphic rating procedures were used to represent judgments of average darkness. Weiss found that the mean graphic rating data were consistent with the averaging model; the interaction was not significant. However, the magnitude estimation data exhibited signiticant interactions. In particular, the bilinear component of the interaction was highly significant, suggesting that the data were more consistent with a multiplicative model than with an additive model.

The essential assumption underlying use of an additive model, such as Equation 1, in describing judgments of averages is that subjects integrate information from multiple stimuli employing quasi-mathematical cognitive operations consistent with their instructions. Weiss' magnitude estimation data appear to be in conflict with this assumption, suggesting that information may be integrated in this task according to a different rule. However, Weiss pointed out that this is not a necessary interpretation. Subjects may have employed the same composition rule in magnitude estimation as in graphic rating, but mapped their judgments onto responses in different ways in the two tasks. This latter interpretation is consistent with the two-stage model of psychophysical judgment (Attneave, 1962; Curtis, Attneave, \& Harrington, 1968).

According to the two-stage model, in estimating magnitude the subject is required to match the central correlate of a stimulus with a corresponding subjective correlate of a number. Attneave (1962) suggested that subjective number may be related to objective number by a nonlinear function, so that the 
usual magnitude estimation function would reflect both an input transformation associated with the sensory system and an output transformation associated with the mapping of perceptions onto a nonlinear response continuum. If the output transformation is described by a power function. the relation between magnitude judgments and subjective scale values would be described by the expression

$$
\mathrm{J}=\mathrm{a} \mathrm{V}^{\mathrm{m}} \text {. }
$$

where $J$ is a magnitude judgment of the stimulus with subjective scale value $V, a$ is a coefticient reflecting the subject's scale unit, and $m$ is the output exponent. equal to the reciprocal of the exponent for subjective number. Assuming also that $V$ is related to the physical measure of the stimulus by a power function, the relation between stimulus and response would be described by the expression

$$
\mathrm{J}=\mathbf{a}^{\prime} \Phi^{\mathrm{km}}
$$

where $\mathbf{k}$ is the parameter of the input, or sensory, function.

A test of the two-stage model was devised by Curtis et al. (1968), who employed magnitude estimates of the differences between stimuli as a basis for separately estimating the exponents $\mathrm{k}$ and $\mathrm{m}$. The product of $k$ and $m$. estimated from difference judgments of lifted weights, was found to closely approximate the exponent of the empirical power function titted to magnitude estimates of single weights. as predicted from the two-stage model (Equation 3). This result has been replicated in several experiments (Curtis, 1970; Curtis \& Rule, 1972; Rule. Curtis. \& Markley. 1970). and also has been shown to hold when parameters were estimated fron magnitude judgments of sums (Curtis \& Fox. 1969). Curtis (1970) and Curtis and Fox (1969) have shown that the discrepancy between magnitude estimation and category ratings could be accounted for on the basis of different output transformations.

If it is assumed that an additive composition rule describes the subjects' integration of stimulus information in judgments of average magnitude, the two-stage model implies that magnitude estimates of averages would be described by the expression

$$
\mathrm{J}_{\mathrm{ij}}=\mathrm{aS}^{\mathrm{m}_{\mathrm{ij}}},
$$

where $S_{i j}$ represents the neighted sum of the scale values for the $i-t h$ and $j$-th stimuli.

$$
S_{i j}=w_{1} V_{i}+w_{2} V_{j} \text {. }
$$

And if $V$ is related to stimulus magnitude by a power function, such that $V=\Phi^{k}$, Equation 4 may be rewritten

$$
\mathrm{f}_{\mathrm{ij}}=\mathrm{a}\left(\mathrm{w}_{1} \Phi_{\mathrm{i}} \mathrm{k}+\mathrm{w}_{2} \Phi_{\mathrm{j}} \mathrm{k}^{\mathrm{m}} .\right.
$$

In the present study, the relation between the two-stage model and the functional measurement model was investigated for magnitude estimation of averages. It should be noted that the additive model is independent of the form of the input transformation(s). so that a functional measurement scaling solution may be appropriate where the two-stage model is not. However, the functional measurement scaling solution does require that the underlying data structure be additive, and additivity would not hold hwere the relation between $J$ and $S$ was nonlinear (e.g. where $\mathrm{m}$ differs from unity). Consequently. the solution is dependent on the sensitivity of the analysis of variance to violations of additivity. Unless the statistical test is sufficiently powerful. the absence of signiticant interactions may be due simply to error variance (Krantz et al., 1971, p. 445).

\section{MAGNITUDE JUDGMENTS OF AVERAGE LOUDNESS}

\section{Method}

Subjects. The nine subjects included one high school student. two undergraduate and four graduate university students, and two nonacademic staff members. All had normal hearing. The subjects were paid $\$ 2$ per session.

Apparatus and Stimuli. Signals were $1.000 \cdot \mathrm{Hz}$ tones of 250 -msec duration, varying in intensity from 35 to $85 \mathrm{~dB}$ in $10 \cdot \mathrm{dB}$ intervals. For judgments of average loudness, these were combined according to a factorial design to produce 36 stimulus pairs. The signals were generated by a pair of General Radio $1304 \mathrm{~B}$ oscillators attenuated by two banks of attenuators controlled by push-button switches. The signal tones were presented monaurally through IDH-49 matched earphones in Grason-Stadler 001 cushions. The trial sequence, controlled by an Iconix timer. consisted of a $250-\mathrm{msec}$ warning light, followed after $1 \mathrm{sec}$ by the two signal tones. separated by a 1 -sec interstimulus interval. The experiment was conducted in an Industrial Acoustics sound-attenuated shamber.

Procedure. Each subject judged both the magnitude of single tones and the magnitude of the average loudness of paired tones. The subjects were tested in five experimental sessions of approximately $35 \mathrm{~min}$ each. During each session. they were presented with tour series of single stimulus judgments and two series of averaging judgments. Presentation of single stimulus and averaging judgments was counterbalanced over days. Rest periods were provided between tasks and between series in the averaging task.

For magnitude estimation of single stimuli, the subjects were presented with 20 random series of the six stimuli. A 55-dB tone was employed as standard, and the subject was instructed to assign the value 10 to its subjective magnitude. In the averaging task. 10 series of the 36 pairs were presented. A standard pair, consisting of the 55- and $65-\mathrm{dB}$ tones, was employed. The standards were presented once at the beginning of each task during each session.

\section{Results}

Functional measurement analysis. Geometric mean judgments of average loudness were calculated for each subject, and the group results were subjected to an analysis of variance. Additivity required a nonsignificant interaction. Following Weiss' (1972) example, the bilinear component and the residual 
were examined for evidence that magnitude estimation might be consistent with a multiplicative model.

The present analysis differed from that of Weiss in an important respect. Weiss used the same error term to test both components of the interaction, namely the Subject by Stimulus 1 by Stimulus 2 interaction. In the present analysis, each component, the bilinear and the residual, was evaluated with the interaction of the subject with the component tested. These error ternis are more appropriate to the model.

For the present data, neither the overall interaction nor either of the two components was significant $(F<1)$. Therefore, according to the rationale of the analysis, these estimates of average loudness were internally consistent, as required by the additive model, and the marginal means should constitute interval scales of subjective loudness. An analysis was performed to evaluate how well the relationship between scale values and sound pressure was approximated by a power function. The expression fitted to this relation was

$$
\mathbf{v}=\mathbf{a} \Phi^{\mathrm{k}}+\mathbf{c} .
$$

The additive constant, $c$, was necessary since $v$ constituted an interval scale at best (i.e., its origin was arbitrary). A least square fit of the scale values for rows and columns (first and second stimuli presented) was provided by

$$
v_{1}=9.38 \Phi \cdot 42+4.95
$$

and

$$
v_{2}=7.67 \Phi \cdot 50+6.18,
$$

respectively, where $\Phi$ denotes the stimulus measure in sound pressure. Plots of these relations are shown in Figure 1. The two estimates of the exponent differ, but they are about as close as one might expect when parameters are estimated from only six scale values.

Geometric mean magnitude estimates of average loudness are plotted as a function of the scale values of the first stimulus in Figure 2. The additive model predicts that the function for each second stimulus

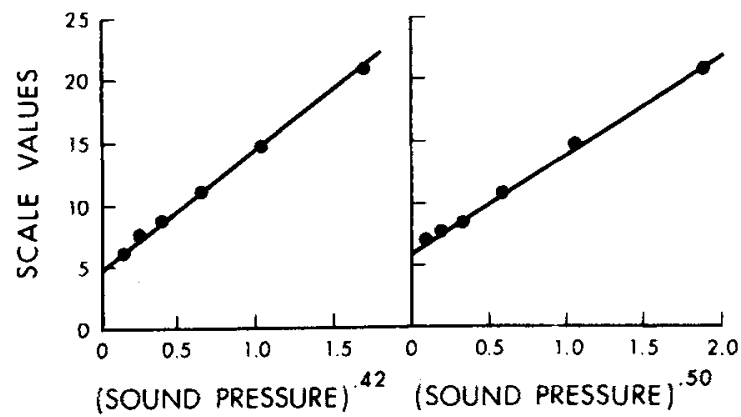

Figure 1. Marginal mean judgments of loudness as a function of sound pressure raised to a power based on a least squares fit.

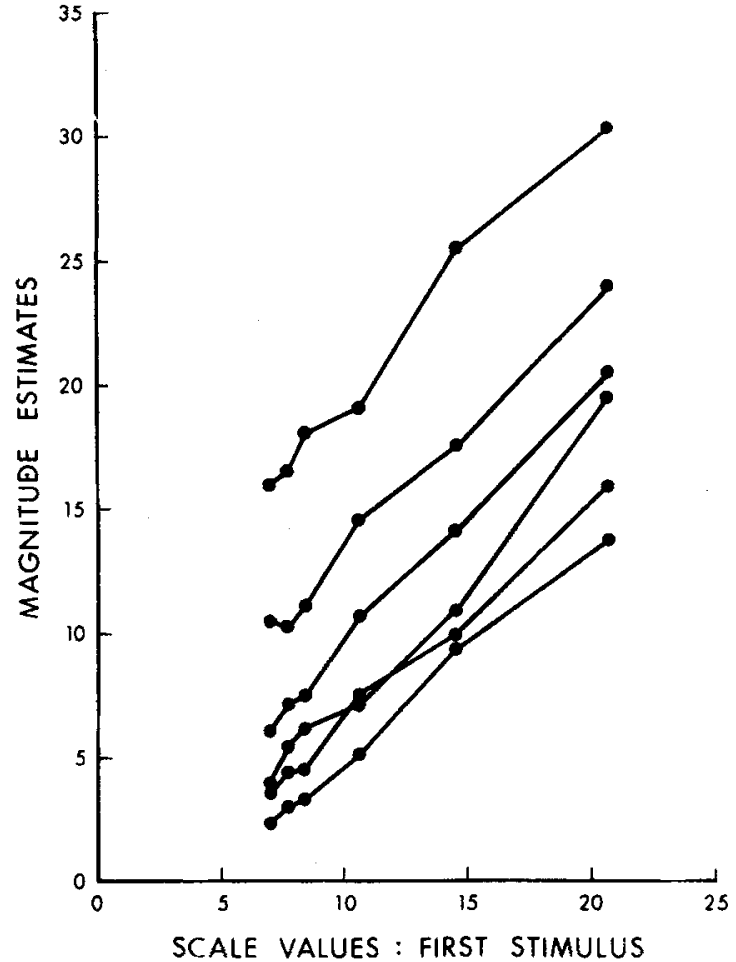

Figure 2. Geometric mean magnitude estimates of average loudness plotted as a function of scale values of the first stimulus. Each curve represents judgments of pairs with a common second stimulus member.

will be linear and that the curves will be parallel. One's initial visual impression suggests that these conditions are satisfied. However, closer inspection and measurement suggests a slight tendency for the functions to diverge as scale values increase. With fallible data such as these, visual inspection alone appears to be insufficient in determining whether or not the functions are in fact parallel.

The two-stage model. According to the two-stage model of magnitude estimation, the curves in Figure 2 should diverge slightly due to a nonlinear output transformation. To evaluate the model, a modified form of Equation 6 was fitted to the group geometric mean judgments of average loudness. The expression fitted was

$$
J_{i j}=\left(a_{1} \Phi_{i} k+a_{2} \Phi_{j}{ }^{k}\right)^{m}+b .
$$

In Equation $8, a_{i}=a^{1 / m_{w h}}$, where a and wh are the coefficient and the weight associated with the first or second stimulus of a pair. The additive constant, $b$, was included because it was not assumed that the origin of the response scale necessarily corresponded to subjective zero. The constant has been found necessary in previous tests of the two-stage model, and justitication for its use has been developed by Rule and Curtis (1973) and Rule, Laye, and Curtis (1974). The parameters of Equation 8 were estimted by an iterative nonlinear least squares solution which weighted each squared deviation by a factor $1 / J_{i j}{ }^{2}$. 
That is, the sum of the relative deviations was minimized. This strategy was employed because the variability of magnitude estimates is known to increase as a function of their magnitudes. The expression minimized has been described elsewhere (Curtis. 1970; Rule et al., 1970).

A solution to Equation 8 was provided by

$$
\mathrm{J}_{\mathrm{ij}}=\left(7.15 \Phi_{\mathrm{i}^{38}}+6.24 \Phi_{\mathrm{j}}{ }^{38}\right)^{1.28}+.54 .
$$

Values of the weights $w_{1}$ and $w_{2}$ were estimated from the relations $w_{1}=a_{1} /\left(a_{1}+a_{2}\right)=.54$ and $w_{2}=$ $a_{2} /\left(a_{1}+a_{2}\right)=.46$. These results suggest that subjects ${ }^{\circ}$ judgments gave approximately equal weighting to the two stimuli of the sequence.

The foregoing equation describes the psychophysical function as a power function with an exponent of .38 , a value smaller than the one which characterized the functional measurement scales. It also implies that subjects' numerical judgments were related to their subjective evaluations of average loudness by a power function with an exponent of 1.28 , a result indicative of a moderately nonlinear output transformation. Such an output function is inconsistent with the results of the analysis of variance. It is consistent, however, with previous findings from studies of magnitude estimation in terms of the two-stage model which have consistently found the output exponent to exceed unity.

A more direct test of the model follows from its prediction of the relation between the parameters $k$ and $\mathrm{m}$ estimated from judgments of average magnitude and the empirical exponent of the power function fitted to magnitude judgments of loudness. The expression fitted to magnitude estimates of loudness was

$$
\mathbf{J}=\mathbf{a} \Phi^{\mathbf{n}}+\mathbf{b},
$$

which includes an additive constant, $b$, as in Equation 8. According to the two-stage model (Equation 3), the empirical exponent $n$ equals the product of the input and output exponents: $n=k m$. This prediction of the model may be tested by comparing the exponent $n$ estimated from judgments of loudness with the product of $k$ and $m$ estimated by fitting Equation 8 to judgments of average loudness. A weighted least squares fit of Equation 9 to magnitude judgments of loudness yielded

$$
J=12.0 \Phi \cdot 44+.08
$$

The exponent of .44 compares favorably with the value of $.48(.38 \times 1.28)$ from the product of $k$ and $m$. However, note also that the mean exponent of the power function fitted to the functional measurement scale was .46, a value even closer to that from magnitude estimation. Consequently, this result does not provide strong support for a nonlinear output transformation.

If the composition rule underlying judgments of average magnitude is. in fact, additive, the foregoing results reflect either upon the validity of the two-stage model or upon the analysis of variance as a test of fit. The two-stage model. of course, incorporates strong assumptions about the forms of the input and output functions. In particular, if the psychophysical function for loudness was not a power function. the apparent nonlinearity in output could be due to error. Alternatively. the analysis of variance may lack sufficient power to detect violations of additivity of the magnitude indicated by an exponent $m$ of 1.28 . For instance, the error term in the analysis of variance may have been inflated by large differences in individual subjects' output functions. Consequently, additional analyses were performed employing nonmetric scaling procedures in an effort to explicate the basis of the discrepancy.

Nonmetric analyses. If judgments of average magnitude reflect some linear combination of subjective scale values, a nonmetric solution to an additive conjoint measurement model provides a basis for recovering initial scales of these values without a priori assumptions concerning the forms of the input and output functions. In the present case, a computer program, UNICON (Note 1), provided scaling solutions for the first and second stimuli of the sequence with a stress of .074 . This stress value indicates that an additive model provides a good fit to the rank order of the data (e.g., some monotone transform of the data). A power function was fitted simultaneously to both sets of scale values, estimating a single exponent but separate coefficients and additive constants. Separate additive constants were required for the two scales because the origins of the scales produced by nonmetric scaling are arbitrary and not necessarily equal. An unweighted least squares solution yielded an exponent $k$ of .37 and coefficients of .98 and .97 . The value of the exponent is almost equal to the value of $k$ obtained by fitting Equation 8 directly to the data. The coefficients reflect the relative weighting of the stimuli of the pairs and suggest that no order effects were present. Figure 3 shows the relation between scale values and sound pressure raised to the power .37. The scales have been corrected for their arbitrary origins. The overall fit is reasonably good, but it is not as good when scales for the tirst and second stimuli are considered separately. The two scales yielded by the functional measurement solution were also found to describe different functions, leaving open the possibility that sequential effects (e.g., time error) may affect the form of the psychophysical function.

The form of the output transformation may be recovered from the relation between judged average loudness and values predicted by the scaling solution. The expression fitted to the data was 


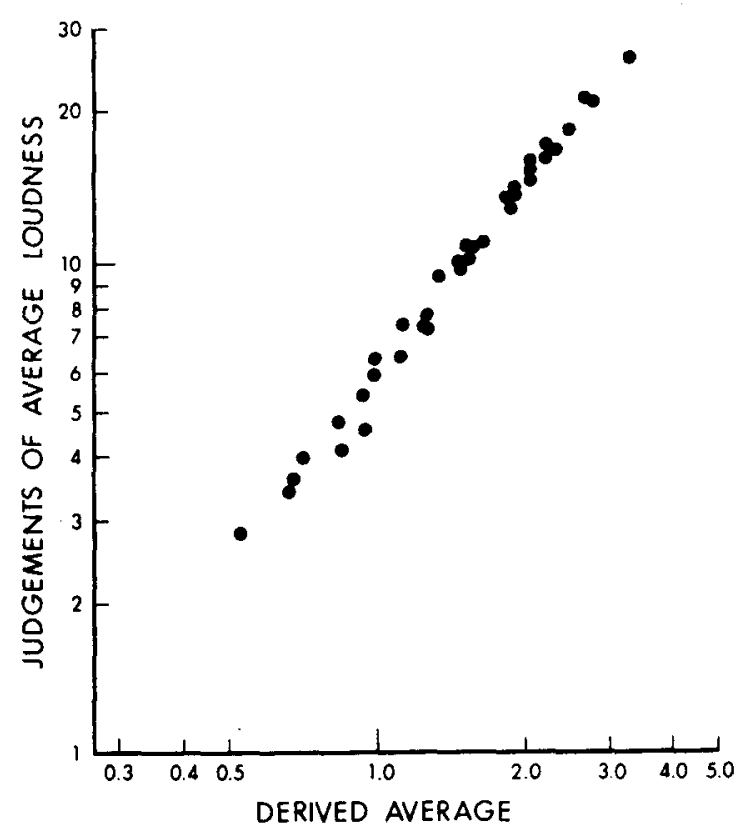

Figure 3. Geometric mean magnitude estimates of average loudness plotted as a function of average loudness predicted from a nonmetric scaling solution.

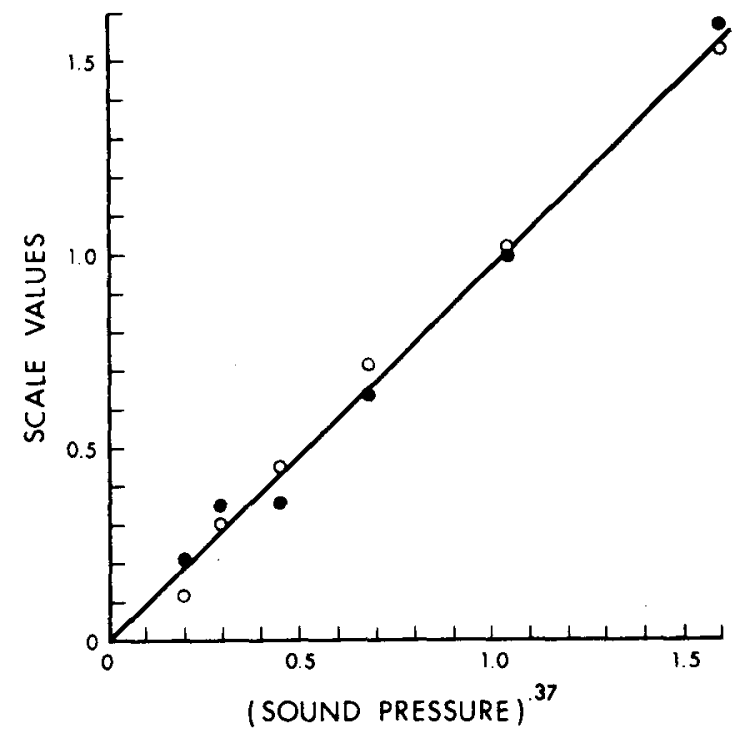

Figure 4. Scale values for loudness from a nonmetric scaling solution as a function of sound pressure raised to the .37 power.

$$
\mathrm{J}_{\mathrm{ij}}=\mathrm{cS}_{\mathrm{ij}}^{\mathrm{m}}+\mathrm{d} \text {, }
$$

where $S$ represents a weighted sum of scale values. A weighted least squares fit was provided by

$$
\mathrm{J}_{\mathrm{ij}}=4.97 \mathrm{~S}_{\mathrm{ij}}{ }^{1.39}+.67 \text {. }
$$

The exponent $\mathrm{m}$ of 1.39 describes an output function even more curvilinear than the one indicated by the analysis directly in terms of the two-stage model. Relaxation of the constraint imposed by assuming the psychophysical relations to be power functions with equal exponents suggests violations of additivity of greater magnitude than is implied by the analysis in terms of the two-stage model. Figure 4 presents a plot in double logarithmic coordinates of $J_{i j}-d$ as a function of $\mathrm{S}_{\mathrm{ij}}$. $\mathrm{J}_{\mathrm{ij}}$ has been corrected for the nonzero additive constant.

Individual differences. The data from individual subjects were subjected to nonmetric parameter estimation, a procedure which combines features of nonmetric scaling with those of iterative least squares solutions to nonlinear equations. A computer program NOPE (Note 2), provided a minimum stress solution for the expression

$$
\mathrm{J}_{\mathrm{ij}}=\mathrm{f}_{\mathrm{mon}}\left(\mathrm{w}_{1} \varnothing_{\mathrm{i}} \mathrm{k}+\mathrm{w}_{2} \varnothing_{\mathrm{j}} \mathrm{k}\right),
$$

where $f_{\text {mon }}$ denotes a weak monotone relation. The procedure provided a unique solution for $k$, and solutions for the relative values of $w_{1}$ and $w_{2}$; i.e., they are unique up to multiplication by a positive constant. The form of the monotone relation $\mathrm{f}_{\text {mon }}$ describes the output function and may be recovered from the relation between the response data and values predicted by the model. Power functions were fitted to this relation. The results of these analyses are presented in Table 1.

Although high stress values indicate that the fit of the model was not very good from some subjects, ordinal violations do not appear to be systematic. Note that estimates of $w_{1}, w_{2}$, and $k$ are consistent over subjects with a single exception, Subject 2 . The estimate of $k$ of .06 for this subject is clearly anomalous. The standard deviation of the estimates is .15 when this subject's exponent is included, but is reduced to .05 when it is excluded. Note also that this subject's output exponent $m$ is exceptionally large. half again as great as that of any other subject. It appears likely that this subject interpreted the instructions in a different way than did the other subjects. The remaining estimates of $k$ are sufficiently similar to suggest that the psychophysical function for loudness may be relatively constant over subjects: i.e..

\begin{tabular}{|c|c|c|c|c|c|c|c|}
\hline \multirow[b]{3}{*}{ Subjects } & \multicolumn{4}{|c|}{$\begin{array}{c}\text { Nonmetric Parameter } \\
\text { Estimation }\end{array}$} & \multirow{2}{*}{\multicolumn{3}{|c|}{$\begin{array}{l}\text { Least Squares } \\
\text { Solution } \\
\mathrm{J}_{\mathbf{i j}}=a S_{i \mathbf{i j}}^{\mathbf{m}}+\mathrm{b}\end{array}$}} \\
\hline & \multicolumn{4}{|c|}{$S_{i j}=f_{\operatorname{mon}}\left(w_{1 \phi l} k+w_{2 \phi j} k\right)$} & & & \\
\hline & $w_{1}$ & $w_{2}$ & $\mathbf{k}$ & Stress & $\mathbf{a}$ & m & b \\
\hline S1 & .50 & .50 & .56 & .11 & 8.71 & .74 & 1.53 \\
\hline S2 & .46 & .54 & .06 & .15 & .01 & 3.04 & -5.27 \\
\hline S3 & .49 & .51 & .48 & .24 & 8.05 & 1.12 & -.54 \\
\hline S4 & .50 & .50 & .53 & .10 & 4.47 & .82 & .26 \\
\hline S5 & .49 & .51 & .42 & .12 & 4.16 & .86 & 1.26 \\
\hline S6 & .48 & .52 & .51 & .21 & 4.72 & 1.60 & .74 \\
\hline S7 & .48 & .52 & .42 & .17 & 2.16 & 1.95 & 3.20 \\
\hline S8 & .47 & .53 & .43 & .16 & 5.05 & 1.82 & 1.06 \\
\hline S9 & .56 & .44 & .41 & .24 & 1.30 & 1.35 & 1.92 \\
\hline Pooled & .50 & .50 & .39 & .07 & 3.95 & 1.25 & .58 \\
\hline
\end{tabular}

Table 1

Parameter Estimates for Individual Subjects 
that their exponents represent independent estimates of the same parameter. If such is the case, the estimates of $.37-.39$ from the pooled data is negatively biased because of the inclusion of Subject 2's data. ${ }^{1}$

Variation on $m$ is considerably greater than that on k. With Subject 2's exponents excluded, the coefficient of variability for $m(s / \bar{m})$ is more than three times as great as the similar statistic for $k$. suggesting that the greater variability on $m$ is not merely a reflection of its larger mean value. If variation on $k$ can be attributed largely to error, it would appear that variation on $\mathrm{m}$ includes a systematic factor as well.

\section{WEISS' JUDGMENTS OF AVERAGE DARKNESS}

Data from judgments of average darkness, reported by Weiss (1972), were subjected to the same analyses as the data from the present study. In Weiss' study, subjects gave magnitude estimates and graphic ratings of the average darkness of pairs of gray papers from the Munsell neutral value series. In his analysis of the magnitude estimation data. Weiss found the bilinear component of the interaction to be significant and the residuals nonsignificant for each of two sessions. The pooled error term was used in all analyses. In our reanalyses, using the Bilinear by Subjects interaction as error term, results were roughly consistent with those of Weiss. For magnitude estimation, the $F$ ratio for the bilinear component was signiticant, but greatly reduced $[\mathrm{F}(1,7)=5.74$, $p<.05]$. The residual was not significant. For the graphic rating data, neither component was significant. These results support Weiss' interpretation that the graphic ratings were consistent with the averaging model, but the magnitude estimation data were not. Power functions fitted to the relation between scale values from the graphic rating data and reflectance yielded exponents of .04 and .16 for the left and right stimulus, respectively. This substantial difference between exponents in an experiment involving simultaneous presentation of stimuli weakens the case for an interpretation of the difference between loudness functions in terms of sequential effects.

In analyzing these data in terms of the two-stage model, the expression fitted to the data was

$$
\mathrm{J}_{\mathrm{ij}}=\mathrm{a}\left(\Phi_{\mathrm{i}} \mathrm{k}+\Phi_{\mathrm{j}}\right)^{\mathrm{m}}+\mathrm{b}
$$

Equation 12 implies that the two stimuli were weighted equally in the judgment of average magnitude, so that the weights were absorbed by the coefficient. In fitting this equation to magnitude estimation data, the sum of squares of relative deviations was minimized, as in the study of loudness averaging. An unweighted solution was obtained in titting the equation to graphic ratings, because the variability of ratings does not, as a rule, increase systematically with increase in response magnitude as is true of magnitude estimations.

Least squares solutions yielded the equations

$$
\mathrm{J}_{\mathrm{ij}}=3915\left(\Phi_{\mathrm{i}}{ }^{21}+\Phi_{\mathrm{j}}{ }^{21}\right)^{-2.04}-146
$$

and

$$
\mathrm{J}_{\mathrm{ij}}=275-73.2\left(\Phi_{\mathrm{i}} \cdot 24+\Phi_{\mathrm{j}} \cdot 24\right) \cdot 75
$$

for the geometric mean magnitude estimates and arithmetic mean graphic ratings, respectively, where the independent variables $\Phi_{i}$ and $\Phi_{j}$ are measured in terms of percent reflectance. The two values of $k$ are very similar in value, suggesting that the same psychophysical function underlies both classes of judgment, a power function with an exponent of $.21-.24$. Both expressions imply that the subjects' response is a monotone decreasing function of the weighted sum of the subjective lightnesses of the stimuli, although the forms of those functions differ. For magnitude estimation the output exponent is negative and the coefficient is positive. whereas for graphic rating the exponent is positive and the coeflicient is negative. This result suggests that subjects have evaluated average loudness in about the same way in the two experiments, but have mapped their evaluations onto responses in different ways. Torgerson (1960) and others have pointed out that magnitude estimation scales of an inverse attribute, such as darkness, tend to be related to the similar scale of the attribute (e.g., lightness) by a reciprocal function. whereas the category scale of the inverse attribute tends to be related to the scale of the attribute by a linear function with a negative slope. Rule, Laye, and Curtis (1974) obtained evidence that in the case of magnitude estimation it is the response scale rather than the perceptual scale that is reversed. The present results tend to confirm this interpretation.

Weiss suggested a different interpretation of the magnitude estimation data based on the results of the analysis of variance. A significant bilinear component of the interaction in association with a nonsignificant residual is consistent with a multiplicative data structure. Weiss pointed out that a multiplicative structure may have resulted from an exponential output transformation, relating this hypothesis to a proposal by Torgerson (1961) to account for the nonlinearity of the relation between magnitude estimates and category judgments. Torgerson's hypothesis was that scales constructed from different classes of response differed in form because instructions forced the subject to treat a single sensory relation as different relations. In his extension of this reasoning to magnitude judgments of averages. Weiss infers that subjects may have been evaluating geometric means. If such were the case, the response 
scale should be consistent with a multiplicative relation with an origin at subjective zero. An analysis of variance was performed on the logarithmically transformed data of Weiss' experiment to evaluate this hypothesis. The results of this analysis did not support the hypothesis. Both the bilinear component and the residual were significant: bilinear, $F(1,7)=$ $63.53, \mathrm{p}<.001 ;$ residual, $\mathrm{F}(15,105)=6.23$, $\mathrm{p}<.001$. An inverse transformation based on the results of the least squares analysis in terms of the two-stage model reduced the bilinear component $[F(1,7)=4.16, p>.05]$, but increased the residual so that it was barely significant $[F(15,105)=2.18$, $\mathrm{p}<.051$.

The foregoing analysis of graphic ratings employed arithmetic means as averages, resulting in an output exponent of .75. In previous studies of category ratings in terms of the two-stage model, Curtis (1970) and Curtis and Fox (1969) obtained linear output functions; however, these investigators employed medians, rather than means, in averaging their data. The use of medians was dictated by the observation that the distribution of ratings tended to be positively skewed at the lower extreme and negatively skewed at the upper extreme of the scale, so that the choice of average might well affect the empirical form of the output transformation. Arithmetic means were used in the present analysis so that results would be comparable to the results of the functional measurement scaling solution. A reanalysis of Weiss' graphic rating data employing median observations yielded the function

$$
\mathrm{J}_{\mathrm{ij}}=220-33.4\left(\varnothing_{\mathrm{i}} \cdot 21+\varnothing_{\mathrm{j}} \cdot 21\right)^{1.14}
$$

The most crucial difference between this equation and the one obtained from arithmetic means is with respect to the output parameter $m$. The value of $m$ of 1.14 denotes a very slightly positively accelerated function, as opposed to the moderately negatively accelerated function obtained from the arithmetic means. and is more similar to the results obtained by Curtis and by Curtis and Fox.

\section{DISCUSSION}

Functional measurement is intended to provide a test of the internal consistency of judgment data and, where consistency holds, to provide a basis for the construction of subjective scales of the stimuli. Two of the sets of data considered in the present study, magnitude estimates of average loudness and graphic ratings of darkness, would be judged internally consistent on the basis of the statistical test. Consequently, the marginal means should provide interval scales of loudness and grayness.

For both sets of data, the relations between marginal means and stimulus measures were well approximated by power functions. Since the interactions were not significant, it would follow that the data should be consistent with the two-stage model where the output exponent approximates unity. When the model was fitted directly to the data, however, in neither instance was this found to be the case. The output exponent from magnitude estimation of average loudness was 1.28 , indicating some violation of additivity (i.e., not additive in terms of the dependent variable). The extent of the nonlinearity indicated by an exponent of this magnitude is relatively modest; however, its effect upon the functional measurement scale is not inconsequential. The average exponent of the power functions fitted to the marginal means was larger than the exponent yielded in terms of the two-stage model: .46 as compared with .38. The inference that output was nonlinear is not dependent on the assumption of the two-stage model that the output function was a power function. Nonmetric scaling, which requires only that the underlying composition rule be additive, leads to the same conclusion.

The difference between the exponents of functions fitted to marginal means and the input exponent $k$ of the two-stage model was more pronounced for Weiss' graphic ratings of darkness. The functional measurement scales yielded a mean exponent of .10 (from exponents of .04 and .16), as compared with a value of $\mathrm{k}$ of .24 from arithmetic mean graphic ratings (or .21 from median ratings). The value of $\mathrm{m}$ for the same data was .75 , indicating a negatively accelerated output function. This result may be compared with the positively accelerated function which characterized magnitude estimation of loudness averages. It is consistent with the usual finding that rating data are nonlinear with magnitude estimation data, and suggests that the nonlinearity is attributable to the differences between the respective output functions.

If these output transformations constitute genuinely systematic violations of additivity, the failure of the analysis of variance to detect them reflects a shortcoming of the functional measurement approach. The insensitivity of the statistical test is most likely to be due to large differences among individual subjects' output transformations which would be reflected in inflated error terms. Results reported by Anderson (1974) appear to be consistent with this interpretation. Anderson reanalyzed data from magnitude estimates of sums of lifted weights reported by Curtis and Fox (1969). The interaction was found to be nonsignificant for the group data, although Curtis and Fox had found the data to be consistent with an additive form of the two-stage model with an output exponent of approximately 1.2. However, in an analysis of individual subjects' data (using the within-cell mean square as error term). Anderson found that for the data of 7 of the 11 subjects the interaction was significant. In other 
words, the modest degree of nonlinearity indicated by the group estimate may have been obscured in the analysis of variance by pooling over the data of individual subjects. many of whose output exponents were distinctly nonlinear, but whose output exponents were highly variable.

Studies employing the two-stage model in studying magnitude estimation have consistently found the exponent of the output function based on group data to be greater than unity; however, the magnitude of the exponent has varied in different studies from 1.14 (Curtis et al.. 1968) to 2.06 (Rule et al. 1974). Individual differences and sampling variability may account for a major part of this variation, although other sources of variability may be associated with experimental parameters (Poulton, 1968). Large individual differences have been observed in other experiments employing the two-stage model (cf. Curtis, 1970; Curtis et al., 1968), and comparable variability has been characteristic of the results of studies of individual magnitude estimation functions (Marks, 1974).

Large individual differences on $m$ are difficult to reconcile with the conception of a nonlinear function for subjective number analogous to the psychophysical functions associated with the sensory attributes: but it is certainly no more difficult than to assume that the differences are attributable to bona fide variation in sensory information processing. It may be that subjects differ $w$ ith respect to the way in which they process numerical information. Some subjects in the nragnitude estimation experiment tend to use numbers much like category labels, employing them to define equal intervals on a subjective scale. Others may respond more directly to the magnitudes implied subjectively by the numbers. It may be significant that the four graduate students who served as subjects in the loudness experiment exhibited strongly nonlinear output functions, whereas the four undergraduate and nonacademic staff subjects tended to exhibit output exponents of approximately unity or snialler. The anomolous subject. Subject 2, was a 16-year-old high school student. However, subject variability with respect to $m$ may also be due to other, idiosyncratic, response factors. Rule and Markley (1971) and, more recently, Duda (1975) have shown that reliable individual differences may be observed in cross-modality matches, as well as in magnitude estimation. In both studies, higher correlations were found between individual exponents for conditions employing a common dependent measure and different stimulus continua than were found for conditions with the same stimulus continuum but different dependent measures.

Of the three sets of data considered in the present study, only Weiss' magnitude estimates of average darkness yielded a significant interaction in the analysis of variance. It should be noted, however, that the analysis in terms of the two-stage model suggests that judgments of average darkness are related to a weighted sum of scale values for lightness by a reciprocal function. Consequently, this significant statistic may have resulted fron Weiss' choice of perceptual attribute. Evidence is available from several studies that magnitude judgments of an inverse attribute (e.g., darkness) are related to judgments of the attribute (e.g.. lightness) by a reciprocal function (Irwin \& Corballis, 1968; Panek \& Stevens, 1966; Stevens \& Guirao, 1962; Stevens \& Harris. 1962; Torgerson. 1960). Exponents of the power functions fitted to the relation between judgments and their related stimulus measures for the attributes and their inverses were found to be approximately equal in magnitude, but the exponents' values for the inverse attributes were negative. Rule, Laye, and Curtis (1974) reported evidence that subjects, in evaluating darkness, evaluated the quantitative relation between stimuli in the same way as in estimating lightness, but used a reciprocal assignment of numbers to their subjective impressions. That is, the values of $k$ for lightness and darkness should be equal and of the same sign. whereas the value for $m$ should be equal in absolute value but should be negative for darkness. Weiss' magnitude estimation data appear to be consistent with this interpretation. Given a reciprocal assignment of numbers to subjective impressions, the relation between judgments of average magnitude and scale values for lightness in terms of the averaging model would be expressed as

$$
\mathrm{J}_{\mathrm{ij}}=\frac{1}{\mathrm{w}_{1} \mathrm{vi}_{\mathrm{i}}+\mathrm{w}_{2} \mathrm{vj}_{\mathrm{j}}}
$$

where $v i$ and $v j$ denote scale values for the $i$-th and $j$-th stimuli and $f$ is the output function.

It is clear that data consistent with Equation 13 are not additive, even if the underlying composition rule is additive and f denotes a linear relation. The reciprocal relation would, of itself, be capable of producing an interaction in the analysis of variance. An additive structure would have resulted from judgments of an inverse attribute only if scale values for darkness had been reciprocally related to scale values for lightness. e.g.. if the subjective scale, rather than the response scale, had been reversed. If such had been the case. judgments of average darkness would have been described by the expression

$$
J_{i j}=f\left(w_{1} u_{i}+w_{2} u_{j}\right),
$$

where $u_{i}$, the darkness scale value of the $i$-th stimulus, was equal to the reciprocal of the scale value for lightness of the same stimulus: $u_{i}=1 / v_{i}$. Neither the findings of Rule et al. nor the present data suggest this to be the case.

Weiss' interpretation of his magnitude estimation data differed from the foregoing. His suggestion was based on the assumption that a significant bilinear 
component of the interaction together with a nonsigniticant residual implies a multiplicative data structure. A multiplicative structure would occur in magnitude estimation where subjects' responses were characterized by an exponential output transformation. Several writers have proposed hypotheses on various theoretical grounds whose consequences imply such a transformation (e.g., Ekman, 1964; Torgerson, 1961; Treisman, 1964). Neither the present data nor evidence from previous studies supports this interpretation. Previous studies employing the two-stage model have obtained output transformations less curvilinear than the exponential, and the results of both magnitude estimation tasks considered in the present study were consistent with these. If the particular hypothesis suggested by Torgerson (1961) had been correct, a logarithmic transformation should have yielded an additive relation and thereby eliminated the significant interaction in the analysis of variance. In fact, the F ratio for the bilinear component was increased and the residual also became significant. What this suggests is that the analysis of variance is not, in itself, a sufficient basis for validating the multiplicative model. With fallible data, results deriving from other nonlinear nodels can produce statistical results indistinguishable from those of multiplicative structures.

Ideally, for a psychophysical scale to be considered valid, it should be both internally consistent with respect to composition rules derived from substantive theory and consistent with independent criteria, criteria external to the data. The value of the functional measurement approach would appear to stem from its role in the former kind of consistency. The present investigation has attempted to consider both. The two-stage model specifies the forms of the input-output relations for judgments of the magnitudes of single stimuli and also specifies the form of that relation where an additive composition rule underlies judgments of the average magnitude of pairs. If the same judgment model is employed in both judgment tasks (e.g., magnitude estimation), so that the data of both should reflect the same output transformation but different composition rules, the model predicts how the two sets of data will be related. Assuming in the present loudness experiment that the exponent of the empirical power function relating magnitude estimates to sound pressure is equal to the product of the input and output transformations, the relation $\mathrm{n}=\mathrm{k} \times \mathrm{m}$ provides a basis for testing the model. The values of $k$ and $m$ estimated by fitting the appropriate form of the model to judgments of average magnitude can be evaluated to determine how well they do, in fact. predict the value of $n$ independently estimated from judgments of apparent loudness. If two tasks require the subject to adopt the same composition rules but different response modes (e.g., magnitude estimation and graphic ratings), the two-stage model also predicts the relation that should hold between the sets of data. The relation should be nonlinear but monotone; the values of the input exponent should be equal, but the output transformations should differ.

\section{REFERENCE NOTES}

1. Roskam, E. E. Unidimensional Conjoint Measurement (UNICON) for multi-faceted designs. Report $74 \mathrm{MA} 09$, Psychologisch Laboratorium, Universiteit, Nijmegen. The Netherlands.

2. The program used was NOPE written in Fortran IV by S. J. Rule, Department of Psychology, University of Alberta.

\section{REFERENCES}

Anderson, N. H. Application of a weighted average model to a psychophysical averaging task. Psychonomic Science, 1967, 8. 227.228.

Anderson, N. H. Averaging model applied to the size-weight illusion. Perception \& Psychophysics, 1970, 8, 1-4. (a)

ANderson, N. H. Functional measurement and psychophysical judgment. Psychological Review, 1970, 77, 153-170. (b)

ANDERSON, N. H. Cross-task validation of functional measurement using judgments of total magnitude. Journal of Experimental Psychology, 1974, 102, 226-233.

Atrneave, F. Perception and related areas. In S. Koch (Ed.), Psychology: A study of a science (Vol. 4). New York: McGraw-Hill, 1962.

Curtis. D. W. Magnitude estimations and category judgments of brightness and brightness intervals: A two-stage interpretation. Journal of Experimental Psychology, 1970, 83, 201-208.

Curtis, D. W., Attneave, F., \& Harrington, T. L. A test of a two-stage model of magnitude estimation. Perception \& Psychophysics, 1968, 3. 25.31.

Curtis, D. W., \& Fox, B. E. Direct quantitative judgment of sums and a two-stage model for psychophysical judgments. Perception \& Psychophysics, 1969, 5, 89-93.

Curtis, D. W., \& Rule, S. J. Magnitude judgments of brightness and brightness difference as a function of background reflectance. Journal of Experimental Psychology, 1972, 95. 215-222.

DudA, P. D. Tests of the psychological meaning of the power law. Journal of Experimental Psychology, 1975, in press.

EKMAN, G. Is the power law a special case of Fechner's law? Perceptual Motor Skills, 1964, 19. 730.

Irwin, R. J., \& Corballis, M. C. On the general form of Stevens' law for loudness and softness. Perception \& Psychophysics, 1968, 3, 137-143.

Krantz, D. H., Luce, R. D., Suppes, P., \& Tversky, A. Foundations of measurement (Vol. 1). New York: Academic Press, 1971.

KrUSKAL, J. B. Multidimensional scaling by optimizing goodness of fit to a nonmetric hypothesis. Psychometrika, 1964, 29. 1-28.

MARKs, L. E. Stimulus range, number of categories, and form of category scale. American Journal of Psychology, 1968, 81, 467-479.

MARKs, L. E. Sensory processes: The new psychophysics. New York: Academic Press. 1974.

Panek, D. W., \& Stevens, S. S. Saturation of red: A prothetic continuum. Perception \& Psychophysics. 1966. 1. 59-66.

Poulton, E. C. The new psychophysics: Six models for magnitude estimation. Psychological Bulletin. 1968, 69,1-19.

Rule, S. J.. \& Curtis, D. W. Reevaluation of two models for judgments of perceptual intervals. Perception \& Psychophysics. 1973, 14, 433-436. 
Rule. S. I.. Curtis. D. W.. \& Markley, R. P. Input and output Iransformations from magnitude estimation. Joumal of Experimental Psychology, 1970. 86. 343-349.

Rule. S. J.. Laye, R. C., \& Curtis, D. W. Magnitude judgments and difference judgments of lightness and darkness. Journal of Experimental Psychology, 1974, 103. 1108-1114.

Rule, S. j., \& Markley. R. P. Subject differences in crossmodality matching. Perception \& Psychophysics. 1971. 9. 115.117.

Stevens. S. S., \& Guirao. M. Loudness, reciprocality, and partition scales. Joumal of the Acoustical Society of America. 1902. 34. 1466-1471:

Stevens. S. S.. \& Harris. J. R. The scaling of subjective roughness and smoothness. Joumal of Experimental Psychology'. 1962. 64. 489.494.

Torgerson. W. S. Quantitative judgment scales. In $H$. Guljiksen and S. Messick (Eds.). Psychological scaling: Theony ard practice. New York: Wiley, 1960.

Torgerson. W. S. Distanices and ratios in psychophysical scaling. Acta Psychologica, 1961, 19, 201-205

Treisman. M. Sensory scaling and the psychophysical law. Quarterly Joumal of Psychology, 1964, 16. 11-22.
Weiss, D. J. Averaging: An empirical validity criterion for magnitude estimation. Perception \& Psychophysics. 1972. 12. 385.388 .

\section{NOTE}

1. Analyses of the data excluding Subject 2 yielded substantially the same results as the conplete data. although values of particular parameters and test statistics differed slightly. Estimates of $\mathbf{k}$ and $\mathbf{m}$ from the eight subjects were .43 and 1.19 . respectively. The predicted empirical exponent from the product of $k$ and $m$ was therefore 51 . which may be compared with an $n$ of .48 estimated fron the loudness judgments. The average exponent of the power functions fitted to the functional measurement scales was also. 48 (from exponents of .44 and .52).

(Received for publication May 12, 1975; revision received July 30,1975 .) 